\title{
Determinação do Parâmetro de Solubilidade de Poliuretanos de PBLH
}

\author{
Eder M. Santos, Marcelo Aguiar, Maria A. F. César-Oliveira , Sônia F. Za \\ wadzki e Leni Akcelrud \\ Laboratório de Polímeros Sintéticos, Departamento de Química, Centro Politécnico, UFPR
}

Resumo: O parâmetro de solubilidade de poliuretanos segmentados foi determinado através de ensaios de inchamento no equilíbrio, tratando-se os dados pela teoria de Flory-Rehner. O segmento flexível dos poliuretanos foi constituído por blocos de oligobutadieno e o segmento rígido foi formado pela reação entre di-isocianato de tolileno e os extensores de cadeia 1,3-propanodiol; 1,4-butanodiol; 1,6-hexanodiol e 2, 2'di-hidroxi-isopropil N, N'anilina. O teor em segmento rígido para os polímeros provenientes dos extensores alifáticos ficou na faixa de $25,1 \%$ a $28,3 \%$, enquanto que, nos polímeros estendidos com o extensor aromático, foi de $32,6 \%$.

Palavras-chave: Poliuretano, polibutadieno líquido hidroxilado, parâmetro de solubilidade.

\section{Solubility Parameter of HTPB Polyurethanes Determination}

Abstract: The solubility parameter of a series of segmented polyurethanes was determined through equilibrium swelling experiments, using the Flory-Rehner theory. The soft segment of the polyurethanes was oligobutadiene and the hard block was formed through the reaction of tolylene diisocyanate with propanediol, butanediol, hexanediol or 2, 2' dihydroxy isopropyl N, N' aniline. The hard segment content in all polymers was about the same for the aliphatic extenders $(25.1 \%$ to $28.3 \%)$ and $32.6 \%$ for the aromatic one.

Keywords: Polyurethane, hydroxyl terminated polybutadiene, solubility parameter.

\section{Introdução}

Os parâmetros de solubilidade $(\delta)$ e de interação polímero - solvente $(\chi)$ são variáveis termodinâmicas de especial interesse na caracterização de sistemas poliméricos tendo em vista que o seu conhecimento permite a determinação da densidade de ligações cruzadas (v) em redes poliméricas simples ou interpenetrantes. O método universalmente adotado para esta determinação baseia-se na teoria de Flory-Rehner e usa dados de inchamento em solventes ${ }^{[1-5]}$. Outros métodos encontram-se também descritos na literatu$\mathrm{ra}^{[6]}$. A relação de Flory-Rehner origina-se da combinação da teoria de Flory-Huggins para misturas polímero-solvente com a teoria da mecânica estatística para a variação da energia livre provocada pelo inchamento $^{[7-12]}$. A equação resultante, descrita abaixo, relaciona o inchamento com a densidade de liga- ções cruzadas em sistemas onde estas se movem simultaneamente e com a mesma velocidade ("affine deformation") durante o inchamento da amostra.

$v=-\left[\ln \left(1-\mathrm{V}_{\mathrm{r}}\right)+\mathrm{V}_{\mathrm{r}}+\chi \mathrm{V}_{\mathrm{r}}{ }^{2}\right] / \rho \mathrm{V}_{1}\left(\mathrm{~V}_{\mathrm{r}}{ }^{1 / 3}-\mathrm{V}_{\mathrm{r}} / 2\right)$

Nesta equação, $v$ é a densidade de ligações cruzadas que corresponde ao número de cadeias efetivo por unidade de volume e é igual a $\rho / \bar{M} c$ sendo $\rho$ a densidade do polímero e $\overline{\mathrm{M}} \mathrm{c}$ o peso molecular médio entre pontos de entrecruzamento. Vr é o volume reduzido (volume da amostra seca/volume da amostra inchada), $\chi$ o parâmetro de interação polímerosolvente e $\mathrm{V}_{1}$ o volume molar do solvente puro.

Este trabalho descreve a determinação do parâmetro de solubilidade de poliuretanos segmentados obtidos a partir de polibutadieno líquido hidroxilado (PBLH), di-isocianato de tolileno (TDI) e como extensores de cadeia 2, 2'-di-hidroxi-isopropil

Autor para correspondência: Leni Akcelrud, LABPOL, Laboratório de Polímeros Sintéticos, Departamento de Química, UFPR, C.P. 19081; CEP: 81531-990, Curitiba,PR. E-mail: akcelrud@quimica.ufpr.br 
N, N'anilina (DHPA); ou 1,3 - propanodiol (PDO); ou 1,4 - butanodiol (BDO); ou 1,6 - hexanodiol (HDO). De posse do valor de $\delta$, foi possível determinar o parâmetro de interação polímero-solvente, $\chi$ e, conseqüentemente, a densidade de ligações cruzadas, $v$, e o $\bar{M}$ c. A funcionalidade maior do que 2,0 do PBLH propicia a formação de ligações cruzadas no segmento flexível.

\section{Experimental}

\section{Reagentes e solventes}

Solventes: acetato de etila, acetona, clorofórmio, diclorometano, metil etil cetona e tetracloreto de carbono foram devidamente tratados e purificados antes de seus usos, conforme recomendado na literatura ${ }^{[13,14]}$. PBLH (Liquiflex P) - Procedência: Petroflex, $\mathrm{PM}=2700 \mathrm{~g} / \mathrm{mol}$; funcionalidade média $=2,2$; foi seco em evaporador rotatório por quatro horas, a $100^{\circ} \mathrm{C}$ (10 mmHg). DHPA - Procedência Dow Chemical; seco em evaporador rotatório por uma hora, a $100^{\circ} \mathrm{C}$ $(10 \mathrm{mmHg})$. TDI - Procedência: Bayer do Brasil S.A. Mistura de isômeros 2,4/2,6, na proporção 80/20. Foi destilado em grade de destilação, a $100^{\circ} \mathrm{C}$ sob vácuo de $10 \mathrm{mmHg}$ (literatura: $\left.126^{\circ} \mathrm{C}, 10 \mathrm{mmHg}{ }^{[15]}\right)$. PDO, BDO e HDO - Procedência: Bayer do Brasil S.A. A metodologia adotada para a purificação dos dióis foi similar. Foram mantidos em hidreto de cálcio por 24 horas e depois destilados sob vácuo de $10 \mathrm{mmHg}$ $\left(\mathrm{T}=83^{\circ} \mathrm{C}\right.$ para o PDO, literatura $=85^{\circ} \mathrm{C}, 10 \mathrm{mmHg}$; $\mathrm{T}=78-80^{\circ} \mathrm{C}$ para o BDO, literatura $=77,5-78^{\circ} \mathrm{C}, 10$ $\mathrm{mmHg} ; \mathrm{T}=135^{\circ} \mathrm{C}$ para o HDO, literatura $=134^{\circ} \mathrm{C}$, $10 \mathrm{mmHg})^{[15]}$.

\section{Técnicas empregadas}

Preparação do poliuretano: As redes elastoméricas foram preparadas em massa, tendo sido utilizada a técnica em duas etapas.

Formação do pré-polímero: O PBLH foi misturado ao TDI de modo a formar um pré-polímero com teor de NCO livre igual a 7\%. A reação foi conduzida a $60^{\circ} \mathrm{C}$, durante 2 horas.

Formação do poliuretano: Ao pré-polímero formado na etapa anterior, foi acrescentado o extensor de cadeia (DHPA, PDO, BDO ou HDO) na temperatura de $100^{\circ} \mathrm{C}$. A quantidade de extensor foi adicionada de modo a manter uma relação $\mathrm{NCO} / \mathrm{OH}$ global igual a 1,1. O meio reacional foi homogeneizado por aproximadamente 15 minutos e submetido ao vácuo para a remoção dos gases dissolvidos na mistura. $\mathrm{O}$ material viscoso assim obtido foi vertido em molde de aço vertical $(25 \times 25 \times 0,3 \mathrm{~cm})$ pré-aquecido. $\mathrm{O}$ conjunto foi levado à estufa para a cura completa por 24 horas, a $100^{\circ} \mathrm{C}$.

Ensaios de inchamento: Das placas obtidas após a cura, foram cortados corpos de prova com dimensões de 2,0 x 2,0 x 0,3 cm. Foram cortados 5 corpos de prova por amostra e por solvente. Os materiais foram pesados e imersos nos solventes selecionados: acetona, clorofórmio, tetracloreto de carbono, acetato de etila, diclorometano e metil etil cetona. $\mathrm{O}$ inchamento foi feito a temperatura ambiente, na ausência de luz, conforme descrito nas normas ASTM 471 ${ }^{[16]}$ e ASTM 1239-55 ${ }^{[17]}$, as quais utilizam a seguinte relação:

$$
\mathrm{S} \%=\left[\left(\mathrm{W}-\mathrm{W}_{\mathrm{o}}\right) / \mathrm{W}_{0}\right] .100
$$

sendo S\% o inchamento no equilíbrio; W o peso final do corpo de prova e $\mathrm{W}_{0}$ o peso inicial.

\section{Resultados e Discussão}

A síntese dos poliuretanos foi feita em processo em duas etapas conforme os esquemas 1 e 2 .

$\mathrm{O}$ excesso de TDI na primeira etapa determina a quantidade de extensor de cadeia a ser usada na segunda. Quanto maior este excesso, maior a quantidade requerida de extensor na etapa posterior e, conseqüentemente, maior o distanciamento entre pontos de entrecruzamento $(\overline{\mathrm{M}} \mathrm{c})$. Esta propriedade caracteriza duas diferenças importantes entre os poliuretanos obtidos a partir de PBLH e os convencionais obtidos a partir de macroglicóis poliéter ou poliéster, cuja reticulação é feita com um extensor trifuncional. Nestes últimos, os pontos de entrecruzamento se localizam nos segmentos rígidos e não na fase flexível e, além disso, a densidade de ligações cruzadas cresce com o teor de segmento rígido, já que estas são introduzidas pelo extensor.

No método do inchamento no equilíbrio o polímero é submetido ao inchamento em vários solventes, escolhidos de forma a cobrir uma faixa de valores de $\delta$. O valor de $\delta$ do polímero $\left(\delta_{2}\right)$ será igual ao do solvente $\left(\delta_{1}\right)$ no qual o inchamento for máximo. De acordo com a teoria ${ }^{[18]}$, a dependência entre o grau de inchamento no equilíbrio de um polímero (Q) em um líquido de baixo peso molecular e o $\delta$ deste líquido é expressa por uma curva de Q (ordenada) 


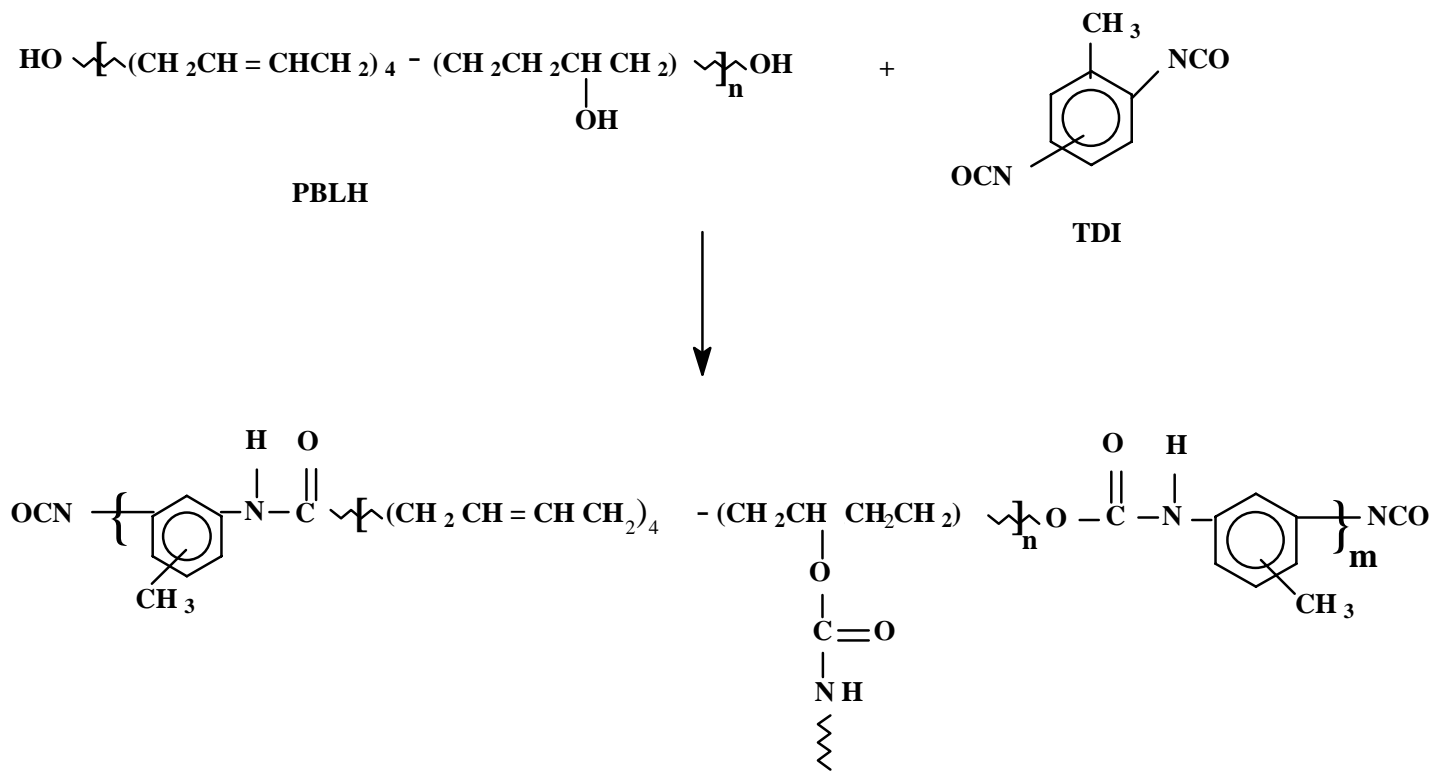

PRÉ-POLÍMERO

Esquema 1. Formação do pré-polímero

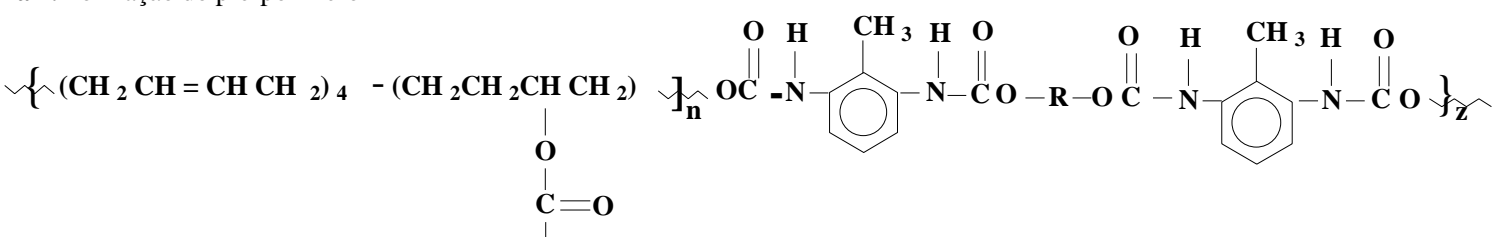

N H

POLIURETANO

$\mathbf{R}=-\left(\mathrm{CH}_{2}\right)_{3}-$

- $\left(\mathrm{CH}_{2}\right)_{4}$ -

$-\left(\mathrm{CH}_{2}\right)_{6}-$

- $\left[\mathrm{CH}\left(\mathrm{CH}_{3}\right) \mathrm{CH}_{2}\right]_{2} \mathrm{~N}-\mathrm{O}$

Esquema 2. Extensão da cadeia

contra $\delta$ (abcissa), a qual passa por um máximo, e cuja equação é a seguinte:

$$
\mathrm{Q}=\mathrm{Q}_{\text {máx }} \exp \left[-\mathrm{V}_{1}\left(\delta_{1}-\delta_{2}\right)^{2}\right]
$$

sendo $\mathrm{V}_{1}$ o volume molar do solvente.

O parâmetro de solubilidade do polímero $\left(\delta_{2}\right)$ será igual ao valor de parâmetro de solubilidade do solvente $\left(\delta_{1}\right)$ correspondente a $Q_{\text {máx }}$. Explicitando $\delta_{2}$ na Equação 3 , temos

$$
\delta_{2}=\delta_{1} \pm\left[\left(1 / \mathrm{V}_{1}\right) \ln \left(\mathrm{Q}_{\text {máx }} / \mathrm{Q}\right)\right]^{1 / 2}
$$

Colocando em gráfico $\left[\left(1 / \mathrm{V}_{1}\right) \ln \left(\mathrm{Q}_{\operatorname{máx}} / \mathrm{Q}\right)\right]^{1 / 2}$ contra $\delta_{1}$, teremos uma reta. No ponto em que esta reta intercepta o eixo da abcissa, $\delta_{1}=\delta_{2}$. Quando o inchamento for máximo $\left(\mathrm{Q}=\mathrm{Q}_{\text {máx }}\right), \delta_{1}$ será igual a $\delta_{2}$.
O grau de inchamento no equilíbrio expresso pelo parâmetro Q é também chamado de coeficiente de inchamento no equilíbrio e é determinado experimentalmente pela relação :

$$
\mathrm{Q}=\left(\mathrm{m}-\mathrm{m}_{\mathrm{o}}\right) / \mathrm{m}_{\mathrm{o}} \cdot \rho
$$

na qual $\mathrm{m}_{\mathrm{o}}$ é a massa do polímero seco, $\mathrm{m}$ a massa do polímero inchado e $\rho$ a densidade do solvente ${ }^{[18]}$.

Os ensaios de inchamento foram realizados em clorofórmio $\left[\delta_{1}=9,30\left(\mathrm{cal} / \mathrm{cm}^{3}\right)^{1 / 2}\right]$, acetato de etila $\left[\delta_{1}=9,05\left(\mathrm{cal} / \mathrm{cm}^{3}\right)^{1 / 2}\right]$, acetona $\left[\delta_{1}=9,77\left(\mathrm{cal} / \mathrm{cm}^{3}\right)^{1 / 2}\right]$, diclorometano $\left[\delta_{1}=9,80\left(\mathrm{cal} / \mathrm{cm}^{3}\right)^{1 / 2}\right]$, metil etil cetona $\left[\delta_{1}=9,22\left(\mathrm{cal} / \mathrm{cm}^{3}\right)^{1 / 2}\right]$ e tetracloreto de carbono $\left[\delta_{1}=8,60\left(\mathrm{cal} / \mathrm{cm}^{3}\right)^{1 / 2}\right]^{[19]}$. A Figura 1 mostra o comportamento típico de um poliuretano estendido com 


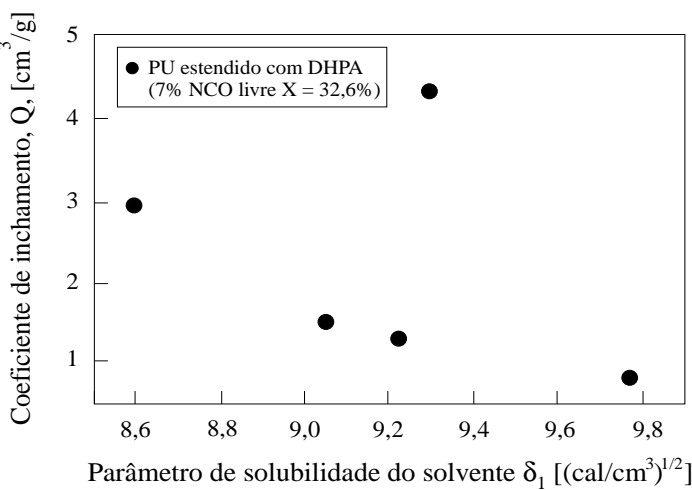

Figura 1. Avaliação do coeficiente de inchamento (Q), para poliuretanos estendidos com DHPA, nos diversos solventes.

DHPA e representa o coeficiente de inchamento máximo Q versus parâmetro de solubilidade do solvente $\delta_{1}$. É possível observar, por inspeção da Figura 1, que o inchamento foi máximo em clorofórmio $\left[\delta_{1}=9,30\left(\mathrm{cal} / \mathrm{cm}^{3}\right)^{1 / 2}\right]$. Este mesmo comportamento foi observado para as demais amostras, com os outros extensores.

Uma vez determinado o solvente no qual o inchamento foi máximo $\left(\mathrm{Q}_{\text {máx }}\right)$, foi possível determinar o valor do parâmetro de solubilidade do poliuretano $\left(\delta_{2}\right)$, conforme descrito anteriormente. A Figura 2 mostra um gráfico de $\left[\left(1 / \mathrm{V}_{1}\right) \ln \left(\mathrm{Q}_{\text {máx }} / \mathrm{Q}\right)\right]^{1 / 2}$, versus $\delta_{1}$, para a obtenção do valor de $\delta_{2}$, no intercepto, tendo sido determinado um valor de 9,12 $\left(\mathrm{cal} / \mathrm{cm}^{3}\right)^{1 / 2}$ para o polímero estendido com DHPA. Para os poliuretanos estendidos com os outros dióis, foi adotada a mesma metodologia, e os valores encontrados estão na Tabela 1 .

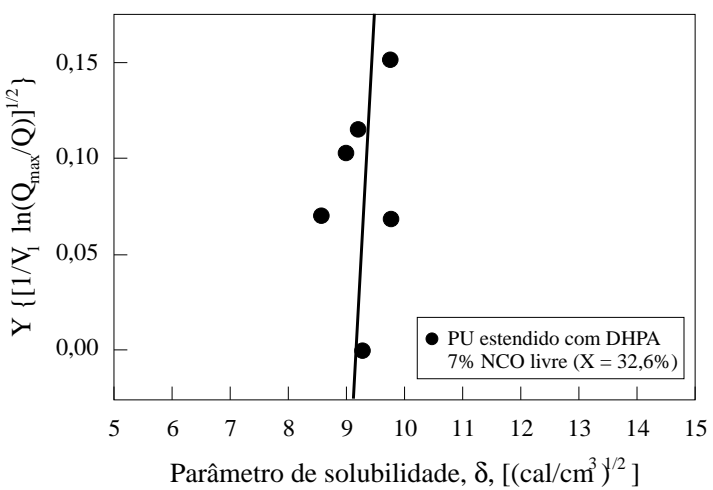

Figura 2. Determinação do valor de $\delta_{2}$ para um poliuretano estendido com DHPA.

O parâmetro de interação polímero-solvente, $\chi$, pode ser decomposto em seus componentes entrópico $\left(\chi_{\mathrm{s}}\right)$ e entálpico $\left(\chi_{\mathrm{h}}\right)$, por ser um parâmetro de energia livre ${ }^{[1,19,20]}$. Assim:

$$
\chi=\chi_{\mathrm{h}}+\chi_{\mathrm{s}}
$$

sendo

$$
\chi_{\mathrm{h}}=\left(\delta_{1}-\delta_{2}\right)^{2} / \mathrm{R} \mathrm{T}
$$

sendo $\mathrm{R}$ a constante dos gases; $\mathrm{T}$ a temperatura absoluta $^{[1,20,21]}$.

Nas condições de inchamento máximo, a contribuição de $\chi_{\mathrm{h}}$ é mínima e o valor do parâmetro de interaçãoé, praticamente igual à contribuição entrópica. A literatura tem usado o valor de 0,34 para $\chi_{\mathrm{s}}{ }^{[22-24]}$.

A partir dos dados de inchamento no equilíbrio, a densidade de ligações cruzadas (v) e o correspondente peso molecular médio entre os pontos de entrecruza-

\begin{tabular}{|c|c|c|c|c|}
\hline & PDO & BDO & HDO & DHPA \\
\hline Teor de segmento rígido, $\mathrm{X}(\%)$ & 25,1 & 26,5 & 28,3 & 32,6 \\
\hline $\begin{array}{l}\text { Porcentagem de absorção no equilíbrio, } \\
\text { S\% }\end{array}$ & 504,78 & 470,45 & 470,02 & 640,56 \\
\hline $\begin{array}{l}\text { Coeficiente de inchamento máximo, } \\
\mathrm{Q}_{\text {máx }}\left(\mathrm{cm}^{3} / \mathrm{g}\right)\end{array}$ & 3,39 & 3,16 & 3,16 & 4,30 \\
\hline Volume reduzido, $\mathrm{Vr}$ & 0,236 & 0,234 & 0,232 & 0,198 \\
\hline Densidade do polímero, $\rho_{2}\left(\mathrm{~g} / \mathrm{cm}^{3}\right)$ & 0,9525 & 1,0394 & 1,0492 & 0,9414 \\
\hline $\begin{array}{l}\text { Parâmetro de solubilidade do } \\
\text { polímero, } \delta_{2}\left(\mathrm{cal} / \mathrm{cm}^{3}\right)^{1 / 2}\end{array}$ & 9,30 & 9,30 & 9,30 & 9,12 \\
\hline $\begin{array}{l}\text { Parâmetro de interação polímero- } \\
\text { solvente, } \chi\end{array}$ & 0,3400 & 0,3400 & 0,3400 & 0,3444 \\
\hline $\begin{array}{l}\text { Densidade de ligações cruzadas, } v \\
(\mathrm{~g} / \mathrm{cm} 3)\end{array}$ & $3,7277 \times 10^{-4}$ & $3,3253 \times 10^{-4}$ & $3,2378 \times 10^{-4}$ & $2,5004 \times 10^{-4}$ \\
\hline $\begin{array}{l}\text { Peso molecular médio entre pontos de } \\
\text { entrecruzamento, } \bar{M} c\end{array}$ & 2600 & 3100 & 3200 & 3800 \\
\hline
\end{tabular}

Tabela 1. Dados obtidos para a determinação de $\overline{\mathrm{M}}$, com a aplicação da teoria de Flory-Rehner. 
mento ( $\overline{\mathrm{M}} \mathrm{c})$ puderam ser calculados, com a aplicação da Equação de Flory-Rehner (Equação 1).

A Tabela 1 mostra os valores dos dados obtidos nos ensaios de inchamento, desde a porcentagem de absorção no equilíbrio, $\mathrm{S} \%$ até a determinação de $\overline{\mathrm{M}} \mathrm{c}$, para os polímeros preparados com $7 \%$ de NCO livre na etapa de pré-polímero. Este teor de NCO livre corresponde a conteúdos em segmento rígido iguais a $25,1 \% ; 26,5 \% ; 28,3 \%$ e $32,6 \%$ para PDO, BDO, HDO e DHPA como extensores, respectivamente.

Apesar do método de inchamento no equilíbrio ser universalmente adotado para a determinação de $v$ em polímeros reticulados, é importante ter em mente que o método pressupõe que as interações intermoleculares sejam unicamente do tipo dispersivo, não levando em consideração interações dipolares ou pontes de hidrogênio. Não obstante, este método tem sido adotado $^{[24-25]}$ quando estas interações estão atuando, efetivamente, como é o caso do polímero utilizado para este trabalho.

Outro ponto ainda que merece consideração é o fato de que os poliuretanos segmentados formam sistemas bifásicos. O parâmetro de interação de um copolímero em bloco em um determinado solvente é, em primeira aproximação, composto de três elementos: os parâmetros de interação de cada bloco com o solvente; e o parâmetro de interação dos blocos entre si. Na maior parte dos poliuretanos, as ligações cruzadas são introduzidas no domínio formado pelo segmento rígido através de um extensor trifuncional, como o trimetilol propano por exemplo. Dados relativos à interação polímero-solvente para um sistema deste tipo (MDI / BDO / PPG 2000)*, usando também inchamento no equilíbrio podem ser encontrados na literatura ${ }^{[26]}$. No caso do poliuretano utilizado neste trabalho, as ligações cruzadas encontram-se no domínio constituído pelo segmento flexível, as quais derivam da funcionalidade média igual a 2,2 do PBLH ${ }^{[27]}$.

Assim, sendo a interação polímero-solvente diferente para cada domínio, os resultados representam um valor médio que reflete a contribuição de cada um deles. A determinação dos componentes espaciais do $\delta$ constitui, ainda, uma outra possibilidade para a seperação das contribuições dos diferentes domíni${ }_{\text {os }}{ }^{[6,25]}$ e é a próxima etapa deste projeto.

* MDI - di - isocianato de 4, 4' difenil metano

BDO - 1, 4 - butanodiol

PPG 2000 - poli propileno glicol de peso molecular 2000

\section{Referências bibliográficas}

1. Akcelrud, L. - Rev. Bras. Eng., 10 (02), p.107 (1993).

2. Panayiotou, C. - Fluid Ph. Equilib., 131 (1-2), p.21 (1997).

3. Abd-El Gawad, N. A. - Bull. Fac. Pharm., 35 (02), p.137 (1997).

4. Zawadzki, S. F.; Sereda, L. \& Akcelrud, L. - Intern. J. Polym. Mater., 33, p.31 (1996).

5. Sen, M. \& Guven, O. - J. Polym. Sci., Pt: B: Polym. Phys., 36 (02), p.213 (1998).

6. Andrade, L.; Amaral, L. \& Akcelrud, L. - Intern. Polym. Mat., 35 (1- 4), p.1 (1997).

7. Huggins, M. L. - J. Chem. Phys., 9, p.440 (1941).

8. Flory, P. J. - J. Chem. Phys., 10, p.51 (1942).

9. Flory, P. J. - "Principles of Polymer Chemistry", Cornell Univ. Press, Ithaca, N.Y. (1953).

10. Flory, P. J. \& Rehner, J. Jr. - J. Chem. Phys., 11, p.512 (1942).

11. Flory, P. J. \& Rehner, J. Jr. - J. Chem. Phys., 11, p.521 (1942).

12. Flory, P. J. - Chem. Revs., 35, p.51 (1944).

13. Morita, T. \& Assumpção, R.M.V. - "Manual de Soluções, Reagentes e Solventes - Padronização, Preparação e Purificação", Edgard Blücher Ed., São Paulo (1972).

14. Perrin, D. D. \& Amarego, W. L. F. - "Purification of Laboratory Chemicals", Pergamon Press, Oxford (1988).

15. Weast, R. C. - "Handbook of Chemistry and Physics", The Chem. Rubber Publ. Co. Cleveland, (1968/1969).

16. American Society for Testing and Materials - ASTM - "Annual Book of Standard", ASTM D 471, p.104 (1979).

17. American Society for Testing and Materials - ASTM - "Annual Book of Standard", ASTM 1239-55, p.175 (1979).

18. Gee, G. - Trans. Faraday Soc., 38, p.269. (1942).

19. Brandrup, J. \& Immergut, E. H. - "Polymer Handbook". John-Wiley, New York (1975). 
20. Sreenevasan, K. - Polym. J., 22 (07), p.620 (1990).

21. Orwoll, R. A. - Rub. Chem. Tech., 50 (03), p.451. (1977).

22. Dudek, T. J.\& Bueche, F. - Rub. Chem. Tech., 37, p.894. (1964).

23. Blanks, R. F. \& Prausnitz, J. M. - Ind. Eng. Chem. Fundam., 3, p.1 (1964).

24. Marco, C.; Bello, A., Fatou, J. G. \& Garza, J. - J. Macromol. Chem., 187, p.177 (1986).
25. Mieczkowski, R - Eur. Polym. J., 28 (1), p.53. (1992).

26. Petrovic, Z. S.; Javni, I.; Divjakovic, V. - J. Polym. Sci., Pt: B: Polym. Phys., 36, p.221 (1998).

27. Zawadzki, S.F.\& Akcelrud, L. - Polym. Intern., 42 (4), p.422 (1997).

Recebido:14/1/00 Aprovado:05/05/00 